\section{New autoimmunity targets}

\section{By Lev Osherovich, Senior Writer}

Apoptotic cell clearance has been posited as a potential strategy to help prevent autoimmunity, but so far it has not been clear how to modulate the process. Now, two research groups have described actual targets to boost the killing and clearance of apoptotic cells, which are the source of many self-antigens.

A study in Nature shows that a soluble form of the proapoptotic Fas ligand (TNF superfamily, member 6) (FASL) promotes an autoimmune disease in mice that resembles systemic lupus erythematosus (SLE). ${ }^{1}$ Another mouse study, reported in Nature Medicine, shows that peroxisome proliferation-activated receptor- $\delta$ (PPARD; PPAR $\delta$ ) combats autoimmunity by boosting the clearance of apoptotic cells by macrophages. ${ }^{2}$

Companies are already targeting FASL and PPAR $\delta$ to treat cancer and metabolic disease, respectively. The challenge in autoimmune disease is to subtly manipulate these targets without eliciting side effects.

\section{Death by membrane}

The FASL study was done by an Australian team led by Andreas Strasser, professor of molecular cancer genetics at The Walter and Eliza Hall Institute of Medical Research. ${ }^{1}$

Multiple molecules of FASL assemble together and engage the Fas receptor (CD95) to trigger apoptosis. Previous work by Strasser and others showed that, in addition to forming various multimers, FASL exists in two distinct forms-secreted and membranebound.

Although mutations in FASL were known to cause autoimmune disease in mice and humans, ${ }^{3}$ it was unclear which form of FASL was responsible for pathology. To answer this question, Strasser's team made knockout mice lacking either of the two FASL isoforms.

Analysis of both types of mice revealed that secreted Fasl interfered with normal proapoptotic signaling by membrane-bound Fasl, which otherwise would prevent autoimmunity by encouraging apoptosis. Strasser thus thinks the secreted form of FASL could be antagonized to reduce autoimmune pathology.

Indeed, cytotoxic T cells from mice lacking secreted Fasl had higher cell-killing activity than wild-type cells, suggesting that secreted FASL interferes with apoptosis. In contrast, T cells from mice lacking the membrane-bound Fasl were worse at cell killing than $\mathrm{T}$ cells from wild-type controls.
Strasser's team got similar results in vivo. Mice lacking secreted Fasl had normal immune responses and survival. Mice with membrane-bound Fasl knockout developed abnormal levels of lymphocytes and autoantibodies and developed an SLE-like autoimmune disease compared with wild-type controls.

"Our work showed that membrane-bound FASL is critical for the killing of target cells and of activated T lymphocytes that would otherwise accumulate and cause lymphadenopathy," which is a swelling of the lymph nodes associated with autoimmune disease, said Strasser.

Altogether, he said, the findings point to "immunosuppressive action by membrane-bound FASL."

Strasser noted that in certain lymphatic cancers, an excess of soluble FASL leads to chronic inflammation and wasting akin to the autoimmune disorder observed in membrane-bound FASL knockout.

"If this is the case, neutralization of secreted FASL by antibodies could prevent wasting," said Strasser.

Strasser's next step is to characterize how soluble FASL promotes autoimmunity and to test whether abnormal autoimmunity triggered by soluble FASL plays a role in tumor development.

Peter Jensen, CEO of TopoTarget A/S, said the contrasting effects of soluble and membrane FASL could result from differences in the multimeric structure of the protein. He suspects that the antiapoptotic effects of secreted FASL could be due to faulty multimerization.

TopoTarget's APO010, a soluble fusion of three human FASL extracellular domains, has completed Phase I testing for cancer.

Even though APO010 is not membrane-bound, Jensen said that it forms a hexamer that promotes cell killing and doesn't elicit autoimmunity.

"If you have the hexameric form of FASL, it's proapoptotic," said Jensen. "But if you have a mix of different multimers, you might have a mixture of effects. If you have a soluble form that's insufficient for cell killing, you could have a problem."

Argenes Inc. is the other biotech with a disclosed FASL program in the clinic. The company's ARG098, a mAb against CD95, is in Phase I to treat rheumatoid arthritis.

Strasser has not patented his findings.

\section{PPAR $\delta$ for the cleanup}

Meanwhile, a Stanford University School of Medicine group found a different way to stimulate apoptosis and reduce autoimmunityagonizing PPAR $\delta .^{2}$

Ajay Chawla, assistant professor of medicine at Stanford, suspected a connection between PPARs and autoimmunity because the nuclear receptors control lipid metabolism and thus could help macrophages digest lipid-rich apoptotic cell debris.

His team found that PPAR $\delta$, but not other PPARs, is critical for the ability of macrophages to dispose of apoptotic cells. In cell culture, macrophages treated with a PPAR $\delta$ agonist had higher levels of surface 


\section{TARGETS \& MECHANISMS}

proteins needed to detect and ingest apoptotic cells than mock-treated control cells.

Conversely, macrophages from mice lacking PPAR $\delta$ had lower levels of activation when exposed to apoptotic cells than macrophages from wild-type controls. In vivo, PPAR $\delta$ deletion led to an SLE-like autoimmune disorder compared with what was seen in wild-type controls.

Chawla thinks PPAR $\delta$ is required for timely digestion of apoptotic cells, which prevents autoimmunity-triggering antigens from escaping into the environment.

"PPAR $\delta$ regulates a set of genes that allow macrophages to detect apoptotic cells," he said. "If you have impairment in the detection of dying cells, you get an autoimmune response."

"We could potentially use PPAR $\delta$ agonists to enhance clearance of apoptotic cells," Chawla said. "The hypothesis is that the overall effect will be to block development of autoimmune responses."

To test this idea, Chawla plans to test PPAR $\delta$ agonists in mouse models of SLE.

First-generation PPAR $\delta$ agonists did not advance far into the clinic, largely due to safety concerns stemming from the receptor's ubiquitous expression and its vital role in lipid metabolism. Indeed, GlaxoSmithKline plc discontinued GW501516, a PPAR $\delta$ agonist related to the one used by Chawla's team, after Phase II testing for dyslipidemia. GSK spokesperson Janet Morgan said that the company recently discovered that GW501516 induced tumors in rodents.

"PPAR $\delta$ is expressed in nearly every cell, so this is an intrinsic limitation" on specifically targeting the pathway in macrophages, said Chawla.

Next-generation PPAR $\delta$ agonists in development include Metabolex Inc.'s MBX8025, which is in Phase II for dyslipidemia, sanofi-aventis Group's SAR351034, which is in Phase I for diabetes and dyslipidemia and Cerenis Therapeutics S.A.'s CER-002, which is in Phase I for atherosclerosis.

Chawla has not patented his findings.

Osherovich, L. SciBX 2(42); doi:10.1038/scibx.2009.1556

Published online Oct. 29, 2009

REFERENCES

1. O'Reilly, L.A. et al. Nature; published online Oct. 1, 2009; doi:10.1038/nature08402

Contact: Andreas Strasser, The Walter and Eliza Hall Institute of Medical Research, Parkville, Victoria, Australia e-mail: strasser@wehi.edu.au

2. Mukundan, L. et al. Nat. Med.; published online Oct. 12, 2009; doi:10.1038/nm.2048

Contact: Ajay Chawla, Stanford University School of Medicine, Stanford, Calif. e-mail: achawla@stanford.edu

3. Rieux-Laucat, F. et al. Science 268, 1347-1349 (1995)

COMPANIES AND INSTITUTIONS MENTIONED

Argenes Inc., Tokyo, Japan

Cerenis Therapeutics S.A., Labége, France

GlaxoSmithKline plc (LSE:GSK; NYSE:GSK), London, U.K.

Metabolex Inc., Hayward, Calif.

sanofi-aventis Group (Euronext:SAN; NYSE:SNY), Paris, France

Stanford University School of Medicine, Stanford, Calif.

TopoTarget A/S (CSE:TOPO), Copenhagen, Denmark

The Walter and Eliza Hall Institute of Medical Research, Parkville, Victoria, Australia 